404

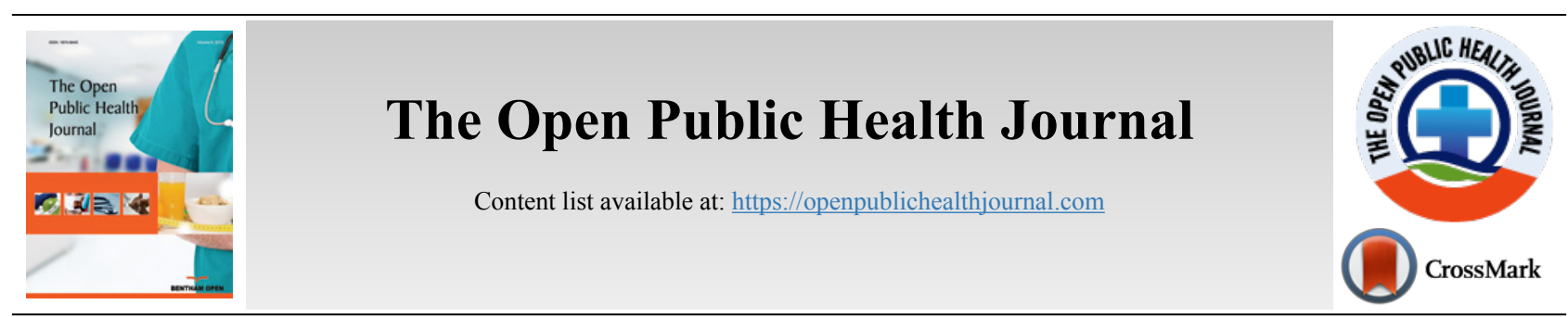

RESEARCH ARTICLE

\title{
COVID-19 Pandemic: A Threat to a Diabetic Patient
}

\author{
Faisal Muhammad ${ }^{1, *}$ \\ ${ }^{\prime}$ Department of Public Health, Faculty of Allied Health Sciences, Daffodil International University, Dhaka, Bangladesh
}

Keywords: COVID-19, Pandemic , Diabetes , Type 2-Diabetes, SARS-CoV-2 , Public Health

\begin{tabular}{|l|l|l|l} 
Article History & Received: May 28, 2020 & Accepted: May 28, 2020 & \\
\hline
\end{tabular}

Coronavirus disease 2019 (COVID-19) is a severe viral disease caused by a newly discovered coronavirus (SARSCoV-2). This disease was declared as a public health emergency by the World Health Organization (WHO) in February 2020. SARS-CoV-2 is the strain of coronavirus that causes COVID-19, and yet the vaccine is not available for this deadly disease. COVID-19 is a kind of contagious disease that is spreading rapidly among older people, and those with preexisting medical conditions, such as diabetes, are at risk of developing more severe symptoms and complications. Patients with Diabetes Mellitus (DM) are susceptible to various infections, more especially the Type-2-Diabetes Mellitus (T2DM) patients. The presence of diabetes complications and fluctuations in the blood glucose levels of diabetic patients makes them harder to treat whenever they develop a viral infection [1].

A recent systematic review and meta-analysis conducted in China reported that diabetes was prevalent in $8.0 \%$ of COVID-19 cases. Other reports from various countries have also verified the presence of diabetes with greater mortality and the need for intensive care during COVID-19 infection. A study was conducted that included the laboratory experiment confirmed COVID-19 patients from two hospitals in Wuhan, China revealed diabetes as the second most common comorbidity among these patients and another study from the same Wuhan, reported that little above $30.0 \%$ of the COVID-19 patients who died, had diabetes. A recent analysis in Italy among deceased COVID-19 patients has revealed diabetes (286/909) as the second most common comorbidity after hypertension. In the late march 2020, Spain reported 5,446 deaths and diabetes was prevalent among $12.0 \%$ of them.

\footnotetext{
* Address correspondence to this author at theDepartment of Public Health, Faculty of Allied Health Sciences, Daffodil International University (DIU) 102 \& 102/1 Shukrabad, Mirpur Road Dhanmondi, Dhaka-1207, Bangladesh;
} Tel: +8801723406483; E-mail: fokkanya@yahoo.com
At the end of March 2020, among the deceased 69 COVID-19 patients in Romania, over half of them had diabetes and cardiovascular diseases [2].

Numerous studies reveal diabetes as a risk factor for the severity of COVID-19 patients. A study of 173 severe COVID-19 patients found that more than $15.0 \%$ of them had diabetes. Usually, diabetes patients have an impaired immune response to infection, and poor glycaemic control impairs several aspects of the immune response to viral infection (including COVID-19) [3]. The data regarding diabetes management during the COVID-19 pandemic are still scarce. However, the Diabetes UK guidance recommends that People with Diabetes (PWD) should follow their normal routine, such as taking a healthy diet, checking feet daily, and physical activity [4]. In the current situation, diabetes management can be burdensome; therefore, extra special attention should be paid. Since, the American Diabetes Association (ADA) gives some suggestions which are essential for diabetes management, such as healthy nutrition (i.e., balanced diet to boost the immune system) and regular physical activity, therefore, it is also recommended that people with diabetes should try to follow the precautions to avoid the deadly virus.

\section{REFERENCES}

[1] International Diabetes Federation (IDF). COVID-19 and Diabetes 2020. Available at: https://www.idf.org/aboutdiabetes/what-is-diabetes /covid-19-and-diabetes.html

[2] Stoian AP, Banerjee Y, Rizvi AA, Rizzo M. Diabetes and the COVID-19 pandemic: how insights from recent experience might guide future management. Metab Syndr Relat Disord 2020 May; 1;18(4): 173-5.

[3] Sten M. Covid-19 infection in people with diabetes. Available at: https://www.touchendocrinology.com/insight /covid-19-infectioninpeople-with-diabetes/.

[4] Hartmann-Boyce J, Morris E, Goyder C, et al. Managing diabetes during COVID-19 pandemic.. 2020. Available at: https://www.cebm.net/covid-19/managing -diabetes-during-thecovid19-pandemic/ 